\title{
The Relationship between CRP at Admission and Thorax CT Findings in Patients Diagnosed with COVID-19
}

\author{
Engin Beydoğan ${ }^{1}$ and Pınar Yürük Atasoy ${ }^{1}$ \\ ${ }^{1}$ Van Training and Research Hospital
}

September 24, 2021

\begin{abstract}
Introduction: The current study aims to evaluate the relationship between C-reactive protein (CRP) levels, thorax CT findings and CT-SS in patients presenting to the emergency department with COVID-19. Methods: Patients diagnosed with COVID-19 by nasopharyngeal rt-PCR $(+)$ in the emergency department were included in the study. In addition to the CRP, ferritin and D-dimer examinations of patients at admission, thorax CT involvement findings and CT-SS results were recorded. The relationship of CRP value with CT-SS and clinical outcome was evaluated. Results: A total of 974 COVID-19 patients, 572 males $(58.7 \%)$ and 402 females (41.3\%), with a mean age of $59.64 \pm 17.34$ years, were included in the study. The CRP values of the patients who needed intensive care and needed respiratory support were also significantly higher at admission (95.1 $\mathrm{mg} / \mathrm{dL}$ vs. $31.05 \mathrm{mg} / \mathrm{dL})(\mathrm{p}<0.001)$. The CRP values of the patients who developed any complications during the treatment of COVID-19 were higher $(79.9 \mathrm{mg} / \mathrm{dL}$ vs. $41.85 \mathrm{mg} / \mathrm{dL})(\mathrm{p}<0.001)$. In the case of CRP $>124.5$, a thorax CT density score 7.35 times higher was determined to be severe. In addition, it was determined that there was a 9.09 -fold increase in the incidence of negative imaging findings in terms of COVID-19 in cases where the CRP value was $<12.5 \mathrm{mg} / \mathrm{dL}$. Conclusion: The CRP levels of COVID-19 patients measured upon admission to the emergency room are correlated with the severity of lung involvement and are an important predictor of clinical outcomes.
\end{abstract}

Title: The Relationship between CRP at Admission and Thorax CT Findings in Patients Diagnosed with COVID-19

\section{ABSTRACT}

Introduction: The current study aims to evaluate the relationship between C-reactive protein (CRP) levels, thorax CT findings and CT-SS in patients presenting to the emergency department with COVID-19.

Methods: Patients diagnosed with COVID-19 by nasopharyngeal rt-PCR $(+)$ in the emergency department were included in the study. In addition to the CRP, ferritin and D-dimer examinations of patients at admission, thorax CT involvement findings and CT-SS results were recorded. The relationship of CRP value with CT-SS and clinical outcome was evaluated.

Results: A total of 974 COVID-19 patients, 572 males (58.7\%) and 402 females (41.3\%), with a mean age of $59.64 \pm 17.34$ years, were included in the study. The CRP values of the patients who needed intensive care and needed respiratory support were also significantly higher at admission $(95.1 \mathrm{mg} / \mathrm{dL}$ vs. $31.05 \mathrm{mg} / \mathrm{dL})$ $(\mathrm{p}<0.001)$. The CRP values of the patients who developed any complications during the treatment of COVID19 were higher $(79.9 \mathrm{mg} / \mathrm{dL}$ vs. $41.85 \mathrm{mg} / \mathrm{dL})(\mathrm{p}<0.001)$.

In the case of CRP $>124.5$, a thorax CT density score 7.35 times higher was determined to be severe. In addition, it was determined that there was a 9.09 -fold increase in the incidence of negative imaging findings in terms of COVID-19 in cases where the CRP value was $<12.5 \mathrm{mg} / \mathrm{dL}$. 
Conclusion: The CRP levels of COVID-19 patients measured upon admission to the emergency room are correlated with the severity of lung involvement and are an important predictor of clinical outcomes.

\section{What's already known about this topic?}

Chest CT is vital in preclinical screening and is highly recommended as a first-line strategy for investigating possible cases of COVID-19.

\section{What does this article add?}

CRP levels and CT-SS increase, the risk in relation to the patient's need for intensive care increases.

\section{Abbreviations}

COVID-19 (coronavirus disease 2019), CT (computed tomography), SARS (severe acute respiratory syndrome), WHO (World Health Organization)

Keywords COVID-19, CRP, Thorax CT, CT-SS, SARS-CoV-2, pandemic

\section{MAIN ARTICLE}

\section{Introduction}

In December 2019, a severe viral pneumonia case series of 41 people, the causative agent of which could not be revealed, was reported in the city of Wuhan, in the Hubei Province of China. ${ }^{1}$ Subsequent whole-genome sequencing and phylogeny analysis showed that SARS-CoV-2 belonged to the betacoronavirus $2 \mathrm{~b}$ lineage, which belongs to the same group as the Severe Acute Respiratory Syndrome coronavirus (SARS-CoV), a highly virulent pathogen in humans. ${ }^{2,3}$ On January 30, 2020, the SARS-CoV-2 infection (COVID-19) was declared a global public health emergency and a pandemic on March 11 by the World Health Organization (WHO). ${ }^{4,5}$

The disease is transmitted by inhalation or contact with infected droplets and the incubation period varies between 2-14 days. Symptoms are usually fever, cough, sore throat, shortness of breath. In most asymptomatic cases, the signs of the disease are mild. However, the disease may progress to pneumonia, acute respiratory distress syndrome and multi-organ dysfunction in some patients (usually the elderly and those with comorbidities). According to recent reports, the death rate from COVID-19 is 5.6-20.3\%, while the mortality rate in severe patients can reach $30-60 \%{ }^{6}$

COVID-19 is mainly diagnosed by reverse transcription-polymerase chain reaction (RT-PCR) to detect SARS CoV-2 nucleic acid in a nasopharyngeal swab (NS) sample. However, due to inappropriate clinical sampling, low patient viral load, and differences in detection rates of different RT-PCR kits, the sensitivity of RTPCR for COVID-19 infection is approximately $71 \% .^{7}$ In addition, direct chest radiographs (x-ray) are less sensitive than thoracic CT, especially in the early stage of COVID-19. ${ }^{8}$ According to current reports, CT can detect the disease before the development of clinical symptoms. ${ }^{9,10}$ Therefore, thorax CT is vital in preclinical screening and is highly recommended as a first-line strategy for investigating possible cases of COVID-19. ${ }^{11}$ In addition, the combination of routine laboratory biomarkers (CRP, LDH, and ferritin \pm Ddimer) can be used for the diagnosis of COVID-19 with an accepted sensitivity and specificity before making a definitive diagnosis by RT-PCR. ${ }^{12}$ However, different results have been reported between CRP levels and the severity of thorax CT involvement, clinical outcomes and disease prognosis in patients diagnosed with COVID-19. ${ }^{13-15}$

This study demonstrates that the increase in CRP levels in correlation with CT-SS in patients with COVID19 presenting to the emergency department successfully predicted adverse clinical outcomes

Materials and Methods

Patients 
Patients who met the inclusion criteria from among those who came to the emergency room with the suspicion of COVID-19 between 01.04.2020 and 31.12.2020 were included in the study. The medical records of the patients included in the study were analyzed through the hospital data processing database. Patients aged 18 years and older who applied to the emergency department of our hospital, for whom the COVID19 diagnosis code (U07.3) was entered according to the ICD-10 classification, were included in the study. Patients younger than 18 years of age, for whom the COVID-19 diagnosis code was not entered, and patients with the COVID-19 diagnosis code but did not have RT-PCR and Thorax CT examination were excluded from the study.

\section{Ethical Committee Approval}

This study was reviewed and approved by the XXXX University Medical Ethics Committee (approval number: XXX). Written and verbal consent forms were obtained from all participants in the study.

\section{Diagnosis of COVID-19 Pneumonia}

A confirmed case of COVID-19 was identified based on the Coronavirus Pandemic Outbreak Method Guide published by the National Health Commission of the Turkish Ministry of Health Science Board. According to this guideline, positive sputum in a nasopharyngeal swab (NS) or endotracheal aspirates (RT-PCR) is accepted as the gold standard in the diagnosis of COVID-19. Disease onset date, clinical classification, RNA test results during hospitalization, and personal demographic information were obtained from clinical records.

Cases with SARS-CoV-2 detected by molecular methods, among the cases suitable for a possible COVID-19 case definition, were included in the study. Patients over 18 years of age and with a hospital stay of 48 hours or more were included in the study. On the contrary, patients who were found to have another infection focus within the first 48 hours were excluded from the study.

\section{Real-Time RT-PCR}

rRT-PCR analysis was performed on materials obtained by NS from patients admitted to the emergency department. A 1-step real-time RT-PCR assay (Bio-Speedy, Turkey) targeting the nucleocapsid gene and open reading frame $1 \mathrm{ab}$ gene was performed with $5 \mu \mathrm{L}$ of total nucleic acid according to the manufacturer's instructions (2B010271500RD, COVID-19 /Flu-RT-qPCR, Bioeksen Ar-GE).

\section{Thorax CT protocol}

CT imaging was performed in the supine position with the arms raised and at the end of inspiration (Toshiba Alexion/Advance, Toshiba Medical Systems Corporation Nashua, Japan). Patients were instructed to hold their breath if clinically possible. Two radiologists experienced in thoracic CT radiology respectively reviewed the thin-section CT images and a decision reached by consensus. Readers identified predominant appearances in CT images such as ground-glass density, crazy-paving pattern, consolidation, and other findings. Both radiologists were unaware of the PCR test results as these were only available after 12-24 hours.

\section{Thorax CT image analysis}

Two experienced radiologists with 11 and 15 years of clinical experience in thoracic CT radiology respectively, reviewed the thin-slice CT images and reached a consensus. They classified the dominant patterns on CT scans as ground-glass opacification (GGO, hazy areas of increasing attenuation that do not block underlying vessels), cobblestone appearance (GGO with interlobular and intralobular septal thickening) and consolidation (homogeneous opacification of the parenchyma). Some other minor findings such as air bronchogram, cavitation, bronchiectasis, pleural effusion, pericardial effusion, pneumothorax and mediastinal lymphadenopathy ( $>1 \mathrm{~cm}$ in short axis diameter) were also recorded in the scans. A pulmonary nodule was defined as a well- or ill-defined round opacity less than $3.0 \mathrm{~cm}$ in diameter. ${ }^{16}$ Pleural effusions were recorded. In the current study, a semi-quantitative CT severity scoring suggested by the RSNA ${ }^{17}$, taking into account the severity of radiological involvement, was calculated separately for 6 lung zones as follows: $1,<0-25 \%$ involvement; 2, 25-50\% involvement; 3, 50-75\% involvement; 4, 75-100\% involvement. The overall CT score was calculated as the sum of the individual zonal scores, and the maximum score was 24 . 


\section{Statistical Analysis}

The SPSS 26.0 (IBM Corporation, Armonk, New York, United States) program was used to analyze the variables. The suitability of the data for normal distribution was evaluated with the Kolmogorov-Smirnov test and the Shapiro-Wilk Francia test. The Mann-Whitney U test was used together with the Monte Carlo results to compare two independent groups with each other according to the quantitative data. The Kruskal-Wallis H Test was used with the Monte Carlo simulation technique to compare more than two groups with each other according to the quantitative data, while the Dunn's Test was used for Post Hoc analyses. The Spearman's rho test was used to examine the correlations of the variables with each other. Sensitivity, specificity and diagnostic accuracy likelihood odds were analyzed and expressed by ROC (Receiver Operating Curve) curve analysis for the relationship between the classification separated by the cut-off value calculated according to the CRP of the groups and the actual classification. Odds ratio values were calculated with $95 \%$ confidence intervals according to these cut-off values. While quantitative variables were expressed as mean (standard deviation) and Median (Minimum / Maximum) and Median (Percentile 25 / Percentile 75) in the tables, categorical variables were shown as n (\%). Variables were analyzed at a $95 \%$ confidence level and a p-value less than 0.05 was considered significant.

\section{Results}

\section{Demographics}

A total of 974 COVID-19 patients, 572 men (58.7\%) and 402 women (41.3\%), with a mean age of $59.64 \pm 17.34$ years, were included in the study. At least one comorbidity was current in $564(58.6 \%)$ of the patients. The distribution of the most common comorbidities was found to be HT (21.2\%), T2DM (15\%), CVD (13.4\%) and COPD $(10.8 \%)$, in order of frequency, similar to that in the literature. The distribution of other comorbidities is summarized in Table 1.

\section{Symptoms}

The most common complaints of patients presenting to the emergency department were found to be shortness of breath $(29.1 \%)$, cough $(21.7 \%)$, fatigue (11.5\%) and fever (11.4\%). However, 111 (5.3\%) patients were found to be asymptomatic (contact). Other application complaints are summarized in Table 2 .

\section{Laboratory Results}

Laboratory tests performed during the patients' admission to the emergency department are summarized in Table 1 . The median CRP value was $56.2(0.26-460) \mathrm{mg} / \mathrm{dL}$, the median ferritin value was 324.65 (61-3130) $\mathrm{mg} / \mathrm{dL}$, and the median D-dimer level was $226(12-43453) \mathrm{mg} / \mathrm{dL}$.

The mean leucocyte level measured at the time of admission of the patients was $7.86 \pm 6.05 \mathrm{cells} / \mathrm{mm} 3$, the lymphocyte count was $1.29 \pm 0.1 \mathrm{~h} / \mathrm{mm} 3$ and the mean neutrophil count was $5.88 \pm 3.83 \mathrm{~h} / \mathrm{mm} 3$. The median NLR value was 4.09 (0.25-71.85) (Table 1) .

\section{Radiological Results}

Thorax CT findings obtained during the patients' admission to the emergency department are summarized in Table 1. Thorax CT findings consistent with typical COVID-19 were detected in 677 patients. While 90 patients had partially significant involvement, 40 patients had involvement consistent with atypical pneumonic infiltrates, 167 patients had negative CT findings for COVID-19 pneumonia despite rt-PCR test positivity. While no signs of involvement were observed on thorax CT in 203 patients (21\%), there were findings consistent with mild lung involvement in 403 patients (41.8\%), moderate in 212 patients (22\%), and severe lung involvement in 145 patients (15\%) (Details inTable 1 ).

\section{COVID-19 Treatment}

Antiviral treatment (Favipiravir) was initiated in all patients because of rt-PCR positivity. In addition, 595 $(33.4 \%)$ of the patients were given additional antibiotic therapy, while $85(4.8 \%)$ were also given convalescent immune plasma therapy (Table 4 ). 


\section{Clinical Outcome}

The median hospital stay of the patients was 8 (1-95) days, while the mean thorax CT-SS was 7.59 \pm 4.2 . It was determined that $307(31.5 \%)$ patients were treated without complications. While the need for intensive care developed in 56 patients (36.9\%), the need for additional respiratory support (NIMV or MV) developed in 290 patients $(29.9 \%)$ during admission or follow-up. A total of $318(32.7 \%)$ patients were diagnosed with severe COVID-19 and mortality developed in 217 (22.3\%) patients (Table 1 ).

Different complications were observed in 298 (30.6\%) patients during the follow-up period after the diagnosis of COVID-19. There was impaired liver function in 153 patients (15.3\%), acute kidney injury in 71 patients $(6.06 \%)$, acute coronary syndrome in 15 patients (1.54\%), atrial fibrillations (AF) in 13 patients (1.13\%), pneumothorax in 10 patients $(1.03 \%)$, and diabetic ketoacidosis developed in 10 patients $(1.03 \%)$. However, while thrombocytopenia and mucosal bleeding, fluid electrolyte imbalance, stroke and acute abdomen were determined in order of frequency, they were at lower rates (Table 3 andTable 4 ).

\section{Relationship between CRP and clinical findings}

CRP values measured at admission were higher in males than females $(73.55$ vs 35.4$)(\mathrm{p}<0.001)$. It was determined that patients with comorbidities had higher CRP values $(70.65$ vs. $41.85 \mathrm{mg} / \mathrm{dL}$ ). The CRP values of the patients, who needed intensive care and needed respiratory support, were also found to be significantly higher at admission $(95.1 \mathrm{mg} / \mathrm{dL}$ vs. $31.05 \mathrm{mg} / \mathrm{dL})(\mathrm{p}<0.001)$ (Table 2) .

While a positive correlation was determined between CRP and patients' ages, the length of hospital stay $(\mathrm{r}=0.118)$, lung density level $(\mathrm{r}=0.445)$, leucocyte count $(\mathrm{r}=0.367)$, neutrophil count $(\mathrm{r}=0.474)$, D-dimer $(\mathrm{r}=0.408)$, and ferritin levels $(\mathrm{r}=0.539)$, there was a negative correlation with lymphocyte levels $(\mathrm{r}=0.367)$. The CRP values of the patients who developed any complications during the treatment of COVID-19 were higher $(79.9 \mathrm{mg} / \mathrm{dL}$ vs. $41.85 \mathrm{mg} / \mathrm{dL})(\mathrm{p}<0.001)$ (Table 2 ).

In the analysis performed to predict the severity of clinical findings and the need for respiratory support, it was determined that a CRP value $>32.6 \mathrm{mg} / \mathrm{dL}$ increased the need for additional respiratory support by 5.05 times. In addition, when the cut-off value of $\mathrm{CRP}>65.95$ was taken, it was determined that there was a 3.81 -fold increase in the risk of death. If the CRP value was $>125 \mathrm{mg} / \mathrm{dL}$, mortality increased significantly (Table 5 ).

\section{Relationship between Thorax CT and CRP}

It was determined that the increase in the severity of involvement in thorax CT was positively correlated with CRP $(\mathrm{p}<0.001)$. Patients with typical COVID-19 thorax CT findings had higher CRP levels compared to those in other patients. If the CRP value was $>124.5$, there was a 7.35 times higher thorax CT density score. In addition, it was determined that there was a 9.09-fold increase in the incidence of negative imaging findings in terms of COVID-19 in cases where the CRP value was $<12.5 \mathrm{mg} / \mathrm{dL}$. The cut-off value for detecting COVID-19 lung involvement with negative imaging findings was determined to be $12.35 \mathrm{mg} / \mathrm{dL}$ (Table 5 ).

\section{Discussion}

The symptoms of symptomatic COVID-19 range widely from mild fever $\left(>37.5^{\circ} \mathrm{C}\right)$ and cough to acute respiratory distress syndrome (ARDS) and death, and the disease follows an unpredictable course. This variability has led to the need for rational use of biomarkers of disease severity and imaging modalities to manage patients appropriately and prevent fatal complications. Based on this, the planned study shows that the increase in CRP levels in patients with a diagnosis of COVID-19 admitted to the emergency department successfully predicts adverse clinical outcomes in correlation with CT-SS.

Many studies have shown that serious illness and death occur in patients with certain risk factors, including advanced age and underlying medical comorbidities. In a case series study of 5,700 patients with COVID-19 infection in New York, the most common comorbidities in hospitalized patients were hypertension (56.6\%), 
obesity $(41.7 \%)$, and diabetes $(33.8 \%) .{ }^{18}$ In addition, a retrospective cohort study of 124 patients with SARS-CoV-2 infection found obesity to be an important risk factor with respect to the need for intubation.

Typical symptoms of COVID-19 are fever, dry cough and fatigue, and in more severe cases, shortness of breath. Less common symptoms include increased sputum, headache, hemoptysis, diarrhea, anorexia, sore throat, chest pain, chills, and nausea and vomiting. ${ }^{1}$ In most people, symptoms appear after an incubation period of 1-14 days (usually about 5 days), and dyspnea and pneumonia develop within an average of 8 days from disease onset. In addition, smell and taste disorders stand out as important symptoms. ${ }^{19}$ In the current study, the most common complaints in patients presenting to the emergency department were dyspnea $(29.1 \%)$, cough $(21.7 \%)$, malaise $(11.5 \%)$, and fever $(11.4 \%)$. In the patient-based evaluation, it was observed that the patients had multiple complaints at the time of their admission and their first complaints were included in the study. However, the fact that 111 (5.3\%) patients were asymptomatic (contact) is also seen as an important finding.

CRP is a nonspecific acute phase protein produced by hepatocytes and is elevated in acute infection or inflammation. ${ }^{20}$ High CRP levels have been observed in COVID-19 patients and are used as an important adjunctive test in triage, diagnosis and predicting prognosis. ${ }^{20,21}$ Elshazli et al ${ }^{22}$ while examining various hematological and immunological markers, emphasized that CRP is a valid biomarker of death from COVID19. It is also thought that the association of higher CRP with worse outcomes may depend on the severity of the disease, which is consistent with the 'cytokine storm' theory of COVID-19, in which the innate immune system is activated by increasing TNF-alpha, IL-6 and IL-1 levels. Studies addressing the clinical utility of CRP have mostly reported a positive association between disease severity and baseline values.

Ali et al. ${ }^{23}$ emphasized that for each unit increase in CRP level, there is a $5 \%$ greater risk that the course of COVID-19 infection in patients will be severe and emphasized that the CRP level can predict a worsening of the disease in non-serious cases. In addition, CRP levels were found to be 10 times higher in patients who died from COVID-19 than those who survived. ${ }^{23,24}$ However, it should be noted that in the review conducted by Ali et al., only studies dealing with the positive relationship between CRP level and disease severity were included. In contrast, other studies have documented no significant differences in CRP levels between mild, severe, and critically ill patients, and sample sizes have been relatively small. ${ }^{25,26}$

In some studies, it has been shown that there are more frequent changes in some laboratory parameters in COVID-19 patients (such as lymphocyte count, CRP, LDH, D-dimer and fibrinogen) ${ }^{27,28}$ Lymphopenia, CRP, LDH, D-dimer and fibrinogen elevation can be used as an auxiliary diagnostic tool in suspected patients with high clinical and thorax CT scanning features, despite a double negative RT-PCR test. ${ }^{29}$ In addition, systemic inflammation as measured by CRP is strongly associated with VTE, AKI, critical illness and mortality in COVID-19. Evaluating the associations between CRP concentrations and respiratory failure requiring mechanical ventilation, patients with a recent $\mathrm{CRP}>5 \mathrm{mg} / \mathrm{dL}$ had an approximately five-fold greater reported risk for acute respiratory distress syndrome (ARDS). ${ }^{30,31} \mathrm{In}$ light of the studies mentioned above, it is clear that high CRP, ESR, IL-6, procalcitonin and serum ferritin levels are associated with worse outcomes and increased mortality in COVID-19 patients. CRP-based approaches to risk stratification and treatment should be tested. ${ }^{32}$

In the current study, CRP levels were high in severe and fatal COVID-19 patients. Patients with severe COVID-19 had significantly higher CRP levels compared to those in patients with non-serious disease [57.9 $(20.9-103.2) \mathrm{mg} / \mathrm{dL}$ vs $33.2(8.2-59.7) \mathrm{mg} / \mathrm{dL}] .{ }^{33}$ In this study, it was determined that the CRP values of the patients, who developed any complications during the treatment of COVID-19, were higher $(79.9 \mathrm{mg} / \mathrm{dL}$ vs. $41.85 \mathrm{mg} / \mathrm{dL})(\mathrm{p}<0.001)$.

A CRP level of $>4 \mathrm{mg} / \mathrm{dL}$ has been shown to be beneficial in the triage of PCR $(+)$ cases presenting with respiratory symptoms/fever [Odds Ratio (OR) 4.75; 95\%, CI 3,28-6,88]. ${ }^{34}$ In most of these studies, CRP with a dual threshold value was used. Recommended values for estimating in-patient mortality ranged from $>10$ $\mathrm{mg} / \mathrm{dL}$ to $>76 \mathrm{mg} / \mathrm{dL}$. In addition to a dual threshold, CRP was also studied in a trichotomized model with two thresholds at $>40 \mathrm{mg} / \mathrm{dL}$ and $>100 \mathrm{mg} / \mathrm{dL} .{ }^{35}$ In the current study, in the analysis performed to predict 
the need for respiratory support according to the severity of clinical findings, it was determined that a CRP value of $>32.6 \mathrm{mg} / \mathrm{dL}$ increased the need for additional respiratory support by 5.05 times. In addition, it was determined that when the CRP value was $>65.95$, there was a 3.81-fold increase in the risk of death, and if the CRP value was $>125 \mathrm{mg} / \mathrm{dl}$, the mortality rate increased significantly.

Recently, with the increase in clinical data, the relationship between liver injury and clinical outcomes of COVID-19 has been further investigated. Ponziani et al.$^{36}$ found that the baseline level of liver enzyme abnormality was associated with an increased risk of intensive care unit admission (OR: 2.19; 1.24-3.89], $\mathrm{p}=0.007$ ). However, further studies have shown that liver enzyme abnormality is an independent predictor of poor prognosis for COVID-19 patients. Yip et al. ${ }^{37}$ found that COVID-19 patients who developed adverse clinical outcomes (including ICU admission, use of invasive mechanical ventilation, and/or death) had a significantly higher incidence of elevated ALT/AST and acute liver injury compared to those who did not (ALT/AST elevation: $70.9 \%$ vs. $19.1 \%, \mathrm{p}<0.001$, acute liver injury: $14.5 \%$ vs. $0.9 \%, \mathrm{p}<0.001$ ). Similarly, Piano et al.${ }^{38}$ showed that liver enzyme abnormality is an independent predictor of ICU admission or death. Therefore, according to the available evidence, it is necessary to regularly monitor the liver functions of patients with COVID-19. In the current study, the highest incidence rate recorded (15.3\%) was that in relation to impaired liver function. Although this condition is often related to the treatments used for the patients, it may also develop secondarily to viral load and hypoxemia.

While the median hospital stay of the patients in this study was 8 (1-95) days, the mean of thorax CT-SS was $7.59 \pm 4.2$. While 307 of the patients recovered without any complications, 349 patients had mild to moderate COVID-19. While the need for intensive care developed in $356(36.9 \%)$ patients, the need for additional respiratory support developed in 290 patients at admission or during follow-up. A total of 318 patients were diagnosed with severe COVID 19 and mortality developed in 217 (22.2\%) patients. In the current study, the significantly higher mortality rate compared to that in the literature was thought to be related to a number of factors, including the fact that our hospital is a tertiary center (as a result of its location within the region), the admission of complicated cases, the evaluation of patients with multiple comorbidities and prolonged stay at hospital after symptom onset.

In a large series of 1,014 patients, Ai et al. ${ }^{39}$ found that thorax CT had a $97 \%$ sensitivity for the diagnosis of COVID-19, while the mean time interval between initial negative and positive RT-PCR was defined as approximately 5 days. Therefore, CT can play a crucial role in the early detection and treatment of COVID19 pneumonia, at least for patients who have been symptomatic for more than three days. ${ }^{40}$ Indeed, $56 \%$ of patients screened within the first 2 days of symptom onset have normal CT findings. Given the important role of thoracic CT, it is important for radiologists to be familiar with the typical CT features associated with this new infection, as well as the imaging criteria for an alternative diagnosis. In our center, where the patients included in the current study were evaluated and the density of the pandemic was quite high, we showed a liberal approach to CT imaging of the thorax in patients with suspected COVID-19 and used the imaging option as early as possible regardless of symptom onset. Therefore, in this study, the time between symptom onset and hospital admission and CT imaging after PCR positivity was detected was very short. This seems to have resulted in negative results with regard to thoracic CT and an increase in the frequency of thorax CT applications even in asymptomatic cases.

In the current study, we aimed to determine the benefit of CRP levels and thorax CT-SS results in determining the clinical outcome and prognosis based on this information. It was determined that patients with typical COVID-19 thorax CT findings had higher CRP levels compared to those of other patients, and thorax CT-SS was positively correlated with CRP $(\mathrm{p}<0.001)$. In addition, it was determined that a cut-off value of $\mathrm{CRP}>124.5 \mathrm{mg} / \mathrm{dL}$ increased the risk of severe involvement of thorax CT-SS 7.35 times, and negative imaging findings in terms of COVID-19 were detected more frequently in cases where the CRP value was $<12.5 \mathrm{mg} / \mathrm{dL}$, and the CRP cut-off value should be $>12.35 \mathrm{mg} / \mathrm{dL}$ to detect COVID-19-related lung involvement in any severe condition. This study demonstrates that the thorax CT severity score constitutes a useful tool for the initial assessment of COVID-19 patients, as it positively correlates with markers of disease severity and offers promising efficacy in predicting critical illness and intensive care unit admissions. 


\section{Limitations}

There are some inevitable limitations in relation to our study, which was conducted using a retrospective file-scanning-based method. Some of these deficiencies are the contact status of the patients, information about the incubation period, and the inability to access medical treatment and examination histories before presentation at the emergency department. In addition, some clinical features were not recorded regularly in some patients in this study, except fever and length of hospital stay. Therefore, only the basic clinical features at presentation, the dynamic change of clinical features, and the relationship between clinical features and CT findings could be analyzed. However, in the current study, the evaluation of a high number of PCR (+) patients with laboratory tests, CT images and clinical outcome characteristics is a strength.

\section{Conclusion}

As a result, as CRP levels and CT-SS increase, the risk in relation to the patient's need for intensive care increases. This information will help guide the management of patients and determine appropriate treatment. CRP levels measured at emergency room admission of COVID-19 patients are correlated with the severity of lung involvement and are an important predictor of clinical outcomes.

\section{Funding}

None.

\section{CRediT authorship contribution statement}

EB: Conceptualization, Methodology, Investigation, Data curation, Writing - original draft, Writing - review \& editing, Visualization, Supervision. PY: Methodology, Investigation, Data curation, Writing - original draft, Writing - review \& editing.

\section{Declaration of Competing Interest}

The authors declare no conflict of interest.

\section{References}

1. Huang C, Wang Y, Li X, et al. Clinical features of patients infected with 2019 novel coronavirus in Wuhan, China. Lancet . 2020;395(10223):497-506. doi:10.1016/S0140-6736(20)30183-5

2. Zhu N, Zhang DY, Wang WL. A novel coronavirus from patients with pneumonia in China, 2019. $N$ Engl Med . 2019;382:727.

3. Lu R, Zhao X, Li J, et al. Genomic characterisation and epidemiology of 2019 novel coronavirus: implications for virus origins and receptor binding. Lancet . 2020;395(10224):565-574. doi:10.1016/S0140$6736(20) 30251-8$

4. World Health Organization (WHO). Coronavirus disease 2019 Situation Report 10 30th January 2020. World Heal Organ . 2020;(January).

5. World Health Organization (WHO). Coronavirus disease 2019 Situation Report 51 11th March 2020. World Heal Organ . 2020;2019(March):2633. doi:10.1001/jama.2020.2633

6. Singhal T. A Review of Coronavirus Disease-2019 (COVID-19).Indian J Pediatr . 2020;87(4):281-286. doi:10.1007/s12098-020-03263-6

7. Fang Y, Zhang H, Xie J, et al. Sensitivity of Chest CT for COVID-19: Comparison to RT-PCR. Radiology . 2020;0(0):200432. doi:10.1148/radiol.2020200432

8. Wong HYF, Lam HYS, Fong AH-T, et al. Frequency and Distribution of Chest Radiographic Findings in Patients Positive for COVID-19.Radiology . 2020;296(2):E72-E78. doi:10.1148/radiol.2020201160 
9. Pan Y, Guan H, Zhou S, et al. Initial CT findings and temporal changes in patients with the novel coronavirus pneumonia (2019-nCoV): a study of 63 patients in Wuhan, China. Eur Radiol . 2020;30(6):33063309. doi:10.1007/s00330-020-06731-x

10. Kim JY, Choe PG, Oh Y, et al. The First Case of 2019 Novel Coronavirus Pneumonia Imported into Korea from Wuhan, China: Implication for Infection Prevention and Control Measures. J Korean Med Sci . 2020;35(5). doi:10.3346/jkms.2020.35.e61

11. Wasilewski P, Mruk B, Mazur S, Półtorak-Szymczak G, Sklinda K, Walecki J. COVID-19 severity scoring systems in radiological imaging - a review. Polish J Radiol . 2020;85(1):361-368. doi:10.5114/pjr.2020.98009

12. Kaftan A, Hussain M, Algenabi A, Naser F, Enaya M. Predictive Value of C-reactive Protein, Lactate Dehydrogenase, Ferritin and D-dimer Levels in Diagnosing COVID-19 Patients: a Retrospective Study. Acta Inform Medica . 2021;29(1):45. doi:10.5455/aim.2021.29.45-50

13. Ai T, Yang Z, Hou H, et al. Correlation of Chest CT and RT-PCR Testing for Coronavirus Disease 2019 (COVID-19) in China: A Report of 1014 Cases. Radiology . 2020;296(2):E32-E40. doi:10.1148/radiol.2020200642

14. Lieveld AWE, Azijli K, Teunissen BP, et al. Chest CT in COVID-19 at the ED: Validation of the COVID-19 Reporting and Data System (CO-RADS) and CT Severity Score: A Prospective, Multicenter, Observational Study. Chest . 2021;159(3):1126-1135. doi:10.1016/j.chest.2020.11.026

15. Tan C, Huang Y, Shi F, et al. C-reactive protein correlates with computed tomographic findings and predicts severe COVID-19 early.J Med Virol . 2020;92(7):856-862. doi:10.1002/jmv.25871

16. Hansell DM, Bankier AA, MacMahon H, McLoud TC, Müller NL, Remy J. Fleischner Society: Glossary of Terms for Thoracic Imaging. Radiology . 2008;246(3):697-722. doi:10.1148/radiol.2462070712

17. Pan F, Ye T, Sun P, et al. Time Course of Lung Changes at Chest CT during Recovery from Coronavirus Disease 2019 (COVID-19).Radiology . 2020;295(3):715-721. doi:10.1148/radiol.2020200370

18. Richardson S, Hirsch JS, Narasimhan M, et al. Presenting Characteristics, Comorbidities, and Outcomes Among 5700 Patients Hospitalized With COVID-19 in the New York City Area. JAMA . 2020;323(20):2052. doi:10.1001/jama.2020.6775

19. Giacomelli A, Pezzati L, Conti F, et al. Self-reported Olfactory and Taste Disorders in Patients With Severe Acute Respiratory Coronavirus 2 Infection: A Cross-sectional Study. Clin Infect Dis . 2020;71(15):889890. doi:10.1093/cid/ciaa330

20. Chen N, Zhou M, Dong X, et al. Epidemiological and clinical characteristics of 99 cases of 2019 novel coronavirus pneumonia in Wuhan, China: a descriptive study. Lancet . 2020;395(10223):507-513. doi:10.1016/S0140-6736(20)30211-7

21. Pepys MB, Hirschfield GM. C-reactive protein: a critical update.J Clin Invest . 2003;111(12):1805-1812. doi:10.1172/JCI18921

22. Elshazli RM, Toraih EA, Elgaml A, et al. Diagnostic and prognostic value of hematological and immunological markers in COVID-19 infection: A meta-analysis of 6320 patients. Afrin F, ed. PLoS One . 2020;15(8):e0238160. doi:10.1371/journal.pone.0238160

23. Ali N. Elevated level of C-reactive protein may be an early marker to predict risk for severity of COVID19. J Med Virol . 2020;92(11):2409-2411. doi:10.1002/jmv.26097

24. Liu F, Li L, Xu M, et al. Prognostic value of interleukin-6, C-reactive protein, and procalcitonin in patients with COVID-19. J Clin Virol Off Publ Pan Am Soc Clin Virol . 2020;127:104370. doi:10.1016/j.jcv.2020.104370 
25. Luo W, Zhang J-W, Zhang W, Lin Y-L, Wang Q. Circulating levels of IL-2, IL-4, TNF- $\alpha$, IFN- $\gamma$, and C-reactive protein are not associated with severity of COVID-19 symptoms. J Med Virol . 2021;93(1):89-91. doi:https://doi.org/10.1002/jmv.26156

26. Chen L, Liu HG, Liu W, et al. [Analysis of clinical features of 29 patients with 2019 novel coronavirus pneumonia]. Zhonghua jie he he hu xi za zhi=Zhonghua jiehe he huxi zazhi=Chinese J Tuberc Respir Dis . 2020;43(0):E005. doi:10.3760/cma.j.issn.1001-0939.2020.0005

27. Liu Y, Yang Y, Zhang C, et al. Clinical and biochemical indexes from 2019-nCoV infected patients linked to viral loads and lung injury.Sci China Life Sci . 2020;63(3):364-374. doi:10.1007/s11427-020-1643-8

28. Tang N, Li D, Wang X, Sun Z. Abnormal coagulation parameters are associated with poor prognosis in patients with novel coronavirus pneumonia. J Thromb Haemost . 2020;18(4):844-847. doi:10.1111/jth.14768

29. Orlacchio A, Gasparrini F, Roma S, et al. Correlations between chest-CT and laboratory parameters in SARS-CoV-2 pneumonia.Medicine (Baltimore) . 2021;100(14):e25310. doi:10.1097/MD.0000000000025310

30. Wu C, Chen X, Cai Y, et al. Risk Factors Associated With Acute Respiratory Distress Syndrome and Death in Patients With Coronavirus Disease 2019 Pneumonia in Wuhan, China. JAMA Intern Med . 2020;180(7):934. doi:10.1001/jamainternmed.2020.0994

31. Goyal P, Choi JJ, Pinheiro LC, et al. Clinical Characteristics of Covid-19 in New York City. $N$ Engl $J$ Med . 2020;382(24):2372-2374. doi:10.1056/NEJMc2010419

32. Smilowitz NR, Kunichoff D, Garshick M, et al. C-reactive protein and clinical outcomes in patients with COVID-19. Eur Heart J . 2021;42(23):2270-2279. doi:10.1093/eurheartj/ehaa1103

33. Qin C, Zhou L, Hu Z, et al. Dysregulation of Immune Response in Patients With Coronavirus 2019 (COVID-19) in Wuhan, China. Clin Infect Dis an Off Publ Infect Dis Soc Am . 2020;71(15):762-768. doi:10.1093/cid/ciaa248

34. Li Q, Ding X, Xia G, et al. Eosinopenia and elevated C-reactive protein facilitate triage of COVID19 patients in fever clinic: A retrospective case-control study. EClinicalMedicine . 2020;23:100375. doi:10.1016/j.eclinm.2020.100375

35. Osuafor CN, Davidson C, Mackett AJ, et al. Clinical Features, Inpatient Trajectories and Frailty in Older Inpatients with COVID-19: A Retrospective Observational Study. Geriatr (Basel, Switzerland) . 2021;6(1). doi:10.3390/geriatrics6010011

36. Ponziani FR, Del Zompo F, Nesci A, et al. Liver involvement is not associated with mortality: results from a large cohort of SARS-CoV-2 positive patients. Aliment Pharmacol Ther . August 2020. doi:10.1111/apt.15996

37. Yip TC-F, Lui GC-Y, Wong VW-S, et al. Liver injury is independently associated with adverse clinical outcomes in patients with COVID-19.Gut . 2021;70(4):733-742. doi:10.1136/gutjnl-2020-321726

38. Piano S, Dalbeni A, Vettore E, et al. Abnormal liver function tests predict transfer to intensive care unit and death in COVID-19.Liver Int . 2020;40(10):2394-2406. doi:10.1111/liv.14565

39. Ai T, Yang Z, Hou H, et al. Correlation of Chest CT and RT-PCR Testing in Coronavirus Disease 2019 (COVID-19) in China: A Report of 1014 Cases. Radiology . February 2020:200642. doi:10.1148/radiol.2020200642

40. Zu ZY, Jiang M Di, Xu PP, et al. Coronavirus Disease 2019 (COVID-19): A Perspective from China. Radiology . 2020;296(2):E15-E25. doi:10.1148/radiol.2020200490

TABLES (1-5)

Table 1. Demographic information 


\begin{tabular}{|c|c|c|c|}
\hline & & & $\mathbf{n}$ \\
\hline \multirow[t]{3}{*}{ Gender } & Gender & & \\
\hline & Female & & 402 \\
\hline & Male & & 572 \\
\hline \multirow[t]{4}{*}{ Lung density } & Lung density & & \\
\hline & Mild involvement & & 408 \\
\hline & Moderate involvement & & 214 \\
\hline & Severe involvement & & 146 \\
\hline \multirow[t]{5}{*}{ Lung radiology } & Lung radiology & & \\
\hline & Typical & & 677 \\
\hline & Partially significant findings & & 90 \\
\hline & Atypical findings & & 40 \\
\hline & Negative & & 167 \\
\hline \multirow[t]{5}{*}{ Course of Disease } & Course of Disease & & \\
\hline & No complication & & 307 \\
\hline & Mild-Moderate course & & 349 \\
\hline & Severe course without mortality & & 101 \\
\hline & Mortal & & 217 \\
\hline \multirow[t]{3}{*}{ Ventilation Support (NIMV or MV) } & Ventilation Support (NIMV or MV) & & \\
\hline & Absent & & 681 \\
\hline & Present & & 290 \\
\hline \multirow[t]{4}{*}{ Intensive Care Unit } & Intensive Care Unit & & \\
\hline & Absent & & 617 \\
\hline & Present & & 356 \\
\hline & $\mathbf{N}$ & & Mean \\
\hline Age & 974 & & 59.64 \\
\hline Length of Hospitalization (Days) & 974 & & 10.87 \\
\hline Chest CTSS & 768 & & 7.59( \\
\hline CRP (mg/dL) & 963 & & 78.69 \\
\hline Leucocyte & 971 & & 7.86 \\
\hline Lymphocyte & 970 & & 1.29 \\
\hline Neutrophil & 966 & & 5.88 \\
\hline NLR & 965 & & 6.55 \\
\hline D-Dimer (mg/dL) & 726 & & 667.67 \\
\hline Ferritin $(\mathrm{mg} / \mathrm{dL})$ & 864 & & 487.78 \\
\hline SD.: Standard Deviation & SD.: Standard Deviation & SD.: Standard Deviation & SD.: $S$ \\
\hline
\end{tabular}

Table 2. Complaints and comorbidities of patients presenting to the emergency department

\section{Complaint}




\section{Comorbidity}

$\mathrm{HT}=$ Hypertension, $\mathrm{T} 2 \mathrm{DM}=$ Type 2 Diabetes Mellitus, $\mathrm{CVD}=$ Cardiovascular disease, $\mathrm{COPD}=$ Chronic obstructive pulmo

Table 3 . Comparison of radiological and clinical findings of COVID-19 patients with CRP

\section{Gender}

Lung density

Lung radiography

Course of Disease

Complication

Comorbidity

Complaint 


\section{Additional respiratory support}

\section{Intensive Care}

Age

Follow-up time

PCR (+) to Discharge (Days)

Chest CT SS

Leucocyte

Lymphocyte

Neutrophil

NLR

D-Dimer $(\mathrm{mg} / \mathrm{dL})$

Ferritin $(\mathrm{mg} / \mathrm{dL})$

? Mann-Whitney U Test (Monte Carlo), ? Kruskal Wallis Test (Monte Carlo), Post Hoc Test: Dunn's Test s Spearman's rh

Table 4. Treatments applied to patients and complications in follow-up

\begin{tabular}{llll}
\hline & & N & \% \\
\hline Treatment & Treatment & & \\
& Antiviral therapy & 973 & $54.6 \%$ \\
& Antibiotherapy (additional) & 595 & $33.4 \%$ \\
& Pulse steroid (additional) & 130 & $7.3 \%$ \\
& Immune plasma therapy & 85 & $4.8 \%$ \\
Complications & Complications & 153 & $15.30 \%$ \\
& Impairment in liver function tests & 71 & $6.06 \%$ \\
& Acute kidney injury & 15 & $1.54 \%$ \\
& Acute coronary syndrome & 13 & $1.13 \%$ \\
& Atrial fibrillation & 10 & $1.03 \%$ \\
& Pneumothorax & 10 & $0.92 \%$ \\
& Diabetic ketoacidosis & 9 & $0.72 \%$ \\
& Bleeding (Thrombocytopenia) & 7 & $0.62 \%$ \\
& Fluid-Electrolyte disorder & 6 & $0.62 \%$ \\
& Stroke & 4 & $0.31 \%$ \\
\hline & Acute abdomen (appendicitis, ileus) & 4 &
\end{tabular}

Table 5. Prediction of CTSS and clinical outcomes by predictive values calculated according to CRP values

\section{CRP}

Need for Additional Respiratory Support

Lung density 


\section{CRP}

Lung radiology

Course of Disease

Roc(Receiver Operating Curve) Analysis (Honley\&Mc Nell - Youden index J ); AUC: Area under the ROC curve; SE: Star 